\title{
Infantile Neuroaxonal Dystrophy
}

National Institute of Neurological Disorders and Stroke (NINDS)

\section{Source}

National Institute of Neurological Disorders and Stroke (NINDS). Infantile Neuroaxonal

Dystrophy Information Page.

Infantile neuroaxonal dystrophy (INAD) is a rare inherited neurological disorder. It affects axons, the part of a nerve cell that carries messages from the brain to other parts of the body, and causes progressive loss of vision, muscular control, and mental skills. While the basic genetic and metabolic causes are unknown, INAD is the result of an abnormal buildup of toxic substances in nerves that communicate with muscles, skin, and the conjunctive tissue around the eyes. Symptoms usually begin within the first 2 years of life, with the loss of head control and the ability to sit, crawl, or walk, accompanied by deterioration in vision and speech. Some children may have seizures. Distinctive facial deformities may be present at birth, including a prominent forehead, crossed eyes, an unusually small nose or jaw, and large, low-set ears. INAD is an autosomal recessive disorder, which means that both parents must be carriers of the defective gene that causes INAD to pass it on to their child. Electrophysiology (nerve conduction velocities) may be helpful for diagnosis, although diagnosis is usually confirmed by tissue biopsy of skin, rectum, nerve or conjunctive tissue to confirm the presence of characteristic swellings (spheroid bodies) in the nerve axons. 